\title{
Internal Photoemission Mechanisms at Interfaces Between Germanium and Thin Metal Films
}

\author{
ERIC Y. CHAN, MEMBER, IEEE, HOWARD C. CARD, MEMBER, IEEE, AND MALVIN C. TEICH, SENIOR MEMBER, IEEE
}

\begin{abstract}
The quantum efficiency associated with the internal photoemission of electrons over the Schottky barrier (of height $\phi_{B}$ ) at the metal-Ge interface has been studied experimentally for several metals ( $\mathrm{Au}, \mathrm{Cu}, \mathrm{Ag}, \mathrm{Pb}$, and $\mathrm{Ni}$ ). A theoretical description of this mechanism has been developed in which we take into account the front and back optical absorptance, hot electron scattering, and multiple reflections of excited electrons from the surfaces of the thin electrode film. We have found it necessary to impose a modification of the Fowler theory of photoemission when applied to internal photoemission from thin metal films over a Schottky barrier. This modification relates to an enhanced photoexcitation within the metal films which is attributed in the present theory to a density of states which exhibits a peaked distribution in energy rather than the simple parabolic bands assumed by Fowlex. It is clear from the present study that the majority of photoelectron excitation occurs from a small region of energy of the order of a fraction of an electron volt near the Fermi energy. The theoretical model presented here defines two important parameters: a hot-electron mean free path $\left(L_{e}\right)$ and an energy $\left(E_{e f}\right)$ given by the difference between the Fermi level and the effective conduction band minimum associated with the region of energy in the metal near the Fermi level where the electron distribution is strongly peaked. Values of $L_{e}$ for $\mathrm{Au}$ is $550 \AA, \mathrm{Ag}$ is $570 \AA, \mathrm{Cu}$ is $450 \AA$, and $\mathrm{Pb}$ is $55 \AA$. $E_{e f}$ for $\mathrm{Au}$ is $0.1 \mathrm{eV}, \mathrm{Ag}$ is $0.152 \mathrm{eV}, \mathrm{Cu}$ is $0.11 \mathrm{eV}, \mathrm{Pb}$ is $0.1 \mathrm{eV}$. The validity of this model is confirmed by the experimental finding that the parameters $L_{e}$ and $E_{e f}$ are independent of metal thickness.
\end{abstract}

\section{INTRODUCTION}

$\mathrm{T}$ HERE has been substantial recent research interest associated with novel detectors operating in the infrared region [1]-[4]. We have presented several modes of photoexcitation in Ge Schottky barriers in previous publications [5][7]. The present paper is concerned with the internal photoemission of electrons from thin metal films over the Schottky barrier $\left(\phi_{B}\right)$ at the metal-Ge interface. Shown in Fig. 1 is the energy band diagram and the corresponding $E-K$ dispersion relation of the metal-Ge Schottky barrier structure. The mechanisms illustrated by arrows 1 and 2 are the band to band transitions of electrons in the Ge substrate. These mechanisms are employed for detection of photons of energy $h \nu>0.67 \mathrm{eV}$, the indirect energy gap of $\mathrm{Ge}$, and are the usual mode of operation for these devices. A full account of the quantum efficiency associated with these band to band processes 1 and 2 is reported in another publication [7]. We discuss here the quan-

Manuscript received September 19, 1979. This work was supported by the Joint Services Electronics Program (U.S. Army, U.S. Navy, and U.S. Air Force) under Contract DAAG29-79-C-0079.

E. Y. Chan was with the Columbia Radiation Laboratory and the Department of Electrical Engineering, Columbia University, New York, NY 10027. He is now with the Western Electric Engineering Research Center, Princeton, NJ.

H. C. Card and M. C. Teich are with the Columbia Radiation Laboratory and the Department of Electrical Engineering, Columbia University, New York, NY 10027.

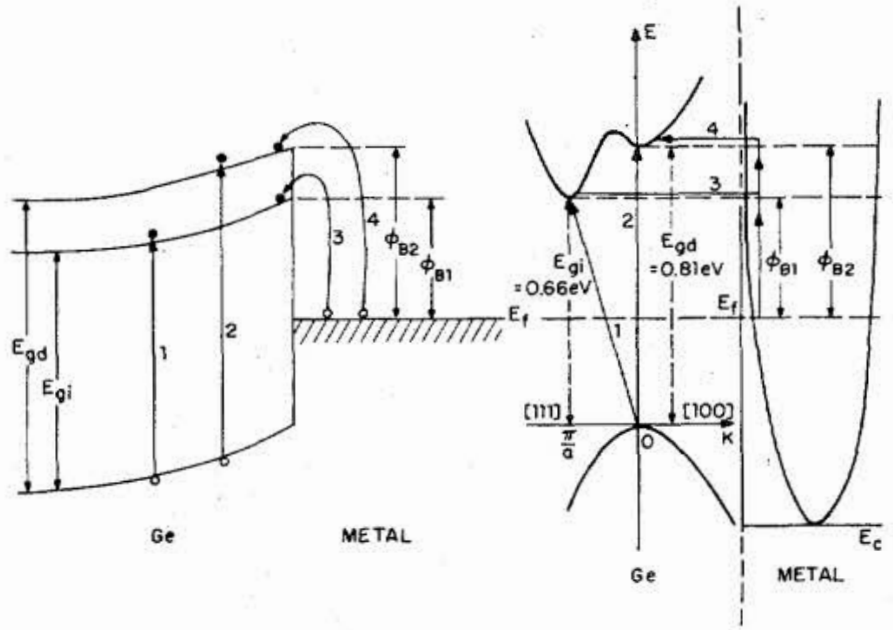

Fig.1. Energy band diagram and dispersion $(E-K)$ relation for metal-Ge Schottky barrier. Arrows $1,2,3$, and 4 indicate the four possible electron transition mechanisms.

tum efficiency associated with the mechanisms indicated by arrows 3 and 4 in Fig. 1 which correspond to the optical excitation of electrons in the metal followed by the scattering of these electrons into the $\mathrm{Ge}$ conduction band. These processes are termed internal photoemission. The standard theory of photoemission from a metal into the vacuum is due to Fowler [8]. This theory assumes that the density of states in the metal is described by the normal parabolic distribution associated with the nearly free electron approximation. Thus in Fowler's theory, the Fermi energy is several electron volts above the conduction band minimum and a single band is considered.

It is known, however (see, for example, [9]), that a realistic description of the density of states in a metal does not follow this simple distribution, but rather shows a distribution with many peaks as a consequence of overlapping bands, nonparabolicity, and deviations in thin metal films from perfect crystalline order. The approach we adopt in the present paper comes to terms with these deviations from ideal behavior by assigning an effective conduction band edge, which is close to the Fermi energy, and which represents the large density of states assumed to exist in the vicinity of the Fermi energy. Thus, we arrive at a modified version of Fowler's theory which is in close agreement with experimental data obtained in this study for internal photoemission from several thin metal films into the conduction band of single crystalline germanium. In addition, the present theory is able to derive quantitative information about the mean free path of photoexcited (hot) electrons with energy well above the Fermi level. 


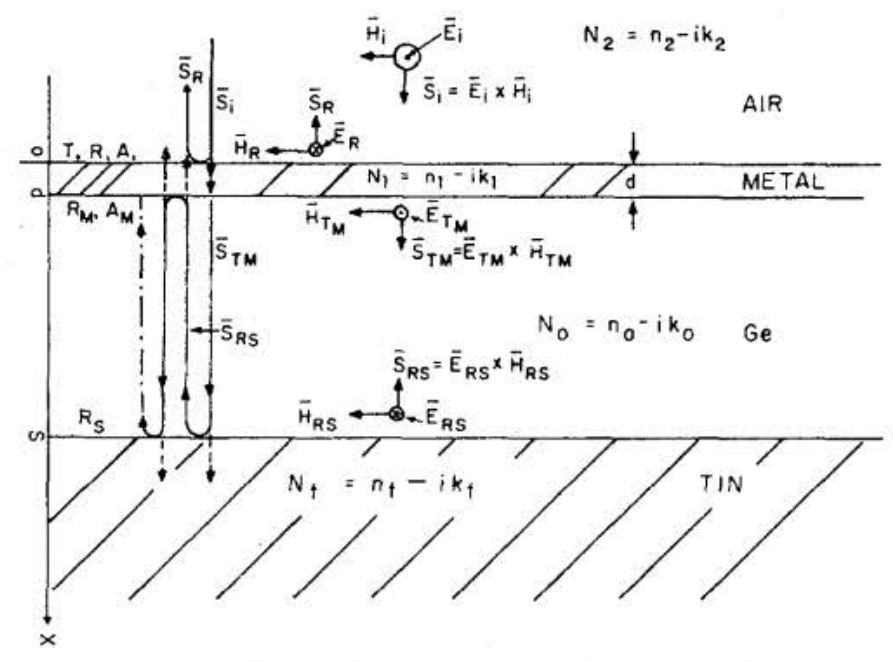

Fig. 2. Schematic diagram for optical properties of metal-Ge Schottky barrier structures. $\bar{E}, \bar{H}$ are electric and magnetic fields of the optical radiation and $\bar{S}$ is the Poynting vector which indicates the direction of power flow. Solid line inside the Ge layer indicates the path for multiple reflection of the transmitted photons. $T, R, A$ are the transmittance, reflectance, and absorptance at the air-metal interface; $R_{M}, A_{M}$ are the reflectance and absorptance at the Ge-metal interface; $R_{S}$ is the reflectance of the thick tin back contact.

\section{Theory of Internal Photoemission}

The external quantum efficiency $\left(\eta_{0}\right)$ of a metal-Ge Schottky barrier associated with internal photoemission can be written as

$$
\eta_{0}=A_{T} F_{e} P_{E}
$$

where $A_{T}$ is the total optical absorptance of the metal electrode, $F_{e}$ is the fraction of the absorbed photons which produce photoelectrons with the appropriate energy and momenta before scattering to contribute to the photocurrent, and $P_{E}$ is the total accumulated probability that one of these photoexcited electrons will be able to overcome $\phi_{B}$ after scattering with cold electrons and with the two boundary surfaces. These three factors are determined as follows.

\section{A. Mathematical Formulation for Optical Absorptance $A_{T}$}

In order to calculate $A_{T}$, the optical properties of the present metal-Ge Schottky barrier structures are schematically represented in Fig. 2. The device is illuminated from the air side with optical radiation that is normally incident. $N_{2}, N_{1}$, $N_{0}$, and $N_{t}$ are the complex indices of refraction of the air, metal, Ge, and the tin back contact. Small $n$ 's are the real part of $N$ 's, small $k$ 's are the extinction coefficients which are the imaginary part of $N$ 's. The respective optical constants ( $n$ 's and $k$ 's) used in the following calculations are summarized in Table $\mathrm{I}$. The optical reflectance $(R)$, absorptance $(A)$, and transmittance $(T)$ are calculated by a two-layer model as follows (see, for example, Heavens [10]):

$$
\begin{aligned}
& T=\frac{n_{0}}{n_{2}} t_{i} t_{i}^{*} \\
& R=r_{i} r_{i}^{*} \\
& A=1-(T+R)
\end{aligned}
$$

TABLE I

Optical Constants used for the Computer Calculation in the SPECIFIED PHOTON ENERgy RANGe

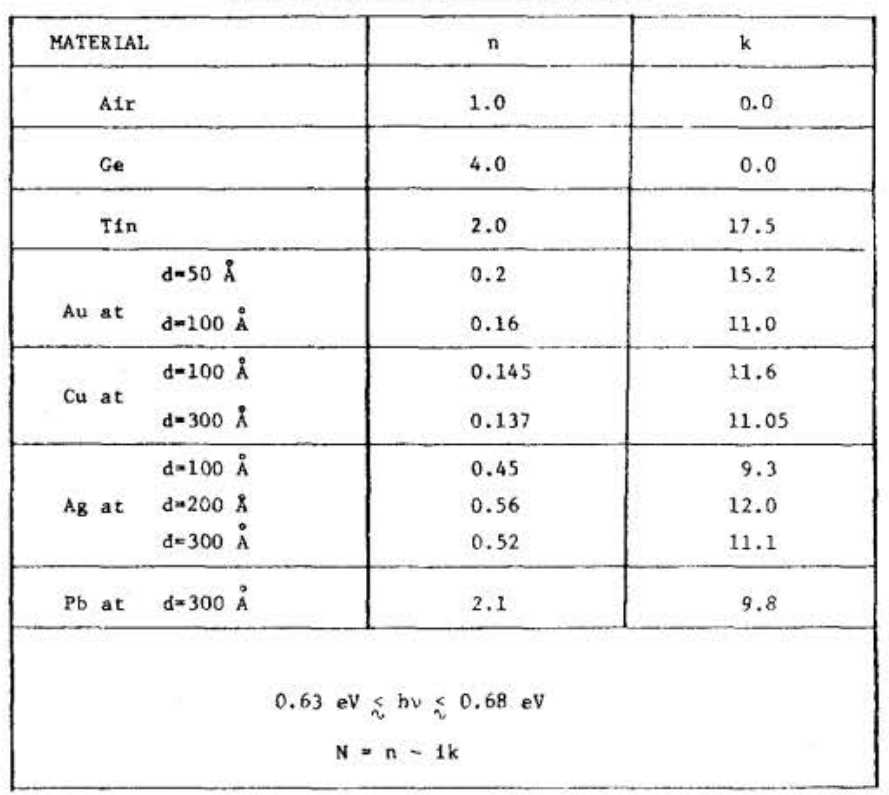

where $r_{i}$ and $t_{i}$ are the reflection and transmission coefficients of the metal-air interface

$$
\begin{aligned}
r_{i} & =\frac{r_{2}+r_{1} \exp \left(-2 i \delta_{1}\right)}{1+r_{1} r_{2} \exp \left(-2 i \delta_{1}\right)} \\
t_{i} & =\frac{t_{1} t_{2} \exp \left(-i \delta_{1}\right)}{1+r_{1} r_{2} \exp \left(-2 i \delta_{1}\right)} \\
\delta_{1} & =\frac{2 \pi}{\lambda} d_{1} N_{1} \\
r_{1} & =\frac{N_{1}-N_{0}}{N_{1}+N_{0}} \\
r_{2} & =\frac{N_{2}-N_{1}}{N_{2}+N_{1}} \\
t_{1} & =\frac{2 N_{1}}{N_{1}+N_{0}} \\
t_{2} & =\frac{2 N_{2}}{N_{1}+N_{2}}
\end{aligned}
$$

and where $\lambda$ is the wavelength of the incident radiation and $d_{1}$ is the thickness of the metal film.

For $h \nu \leqslant 0.66 \mathrm{eV}$, the Ge substrate can be assumed to be transparent to the incident radiation. These transmitted photons in the Ge are multiply reflected between the metal and the tin back contact as indicated in Fig. 2. We define $T_{M}$, $R_{M}$, and $A_{M}$ as the transmittance, reflectance, and absorptance within the metal, of photons incident on the metal film from the germanium substrate; again, using a two-layer calculation we have that

$$
\begin{aligned}
T_{M} & =\frac{n_{2}}{n_{0}} t_{b} t_{b}^{*} \\
R_{M} & =r_{b} r_{b}^{*}
\end{aligned}
$$




$$
\begin{aligned}
A_{M} & =1-\left(T_{M}+R_{M}\right) \\
r_{b} & =\frac{r_{0}+r_{10} \exp \left(-2 i \delta_{1}\right)}{1+r_{10} r_{0} \exp \left(-2 i \delta_{1}\right)} \\
t_{b} & =\frac{t_{0} t_{10} \exp \left(-i \delta_{1}\right)}{1+r_{0} r_{10} \exp \left(-2 i \delta_{1}\right)} \\
r_{10} & =\frac{N_{1}-N_{2}}{N_{1}+N_{2}} \\
r_{0} & =\frac{N_{0}-N_{1}}{N_{0}+N_{1}} \\
t_{10} & =\frac{2 N_{1}}{N_{1}+N_{2}} \\
t_{0} & =\frac{2 N_{0}}{N_{0}+N_{1}} .
\end{aligned}
$$

The reflectance $\left(R_{t}\right)$ of the thick tin back contact can be approximated by a one-layer calculation as follows:

$$
\begin{aligned}
r_{t} & =\frac{N_{0}-N_{t}}{N_{0}+N_{t}} \\
R_{t} & =r_{t} r_{t}^{*} \simeq 90 \text { percent. }
\end{aligned}
$$

The additional optical absorptance $\left(A_{B}\right)$ of the metal film due to multiple scattering of the photons originally transmitted into the $\mathrm{Ge}$ substrate is given as

$$
\begin{aligned}
A_{B}= & T R_{t} A_{M}+T R_{t}^{2} R_{M} A_{M}+R T_{t}^{3} R_{M}^{2} A_{M} \\
& +T R_{t}^{4} R_{M}^{3} A_{M}+\cdots \\
= & T A_{M} R_{t}\left[1+R_{t} R_{M}+\left(R_{t} R_{M}\right)^{2}+\left(R_{t} R_{M}\right)^{3}+\cdots\right] \\
= & T A_{M} R_{t}\left(\frac{1}{1-R_{t} R_{M}}\right) \\
\therefore A_{T}= & A+A_{B} .
\end{aligned}
$$

\section{B. Mathematical Formulation for $F_{E}$}

The nearly free electron gas in a metal obeys Fermi-Dirac statistics; the number of electrons per unit volume having velocity components in the range $u, u+d u, v, v+d v$, and $w$, $w+d w$ ( $u$ normal to metal-Ge interface) is given by the wellknown formula used in the standard Fowler theory [8]

$$
n(u, v, w) d u d v d w=2\left(\frac{m}{h}\right)^{3} \frac{d u d v d w}{e^{\left[1 / 2 m\left(u^{2}+v^{2}+w^{2}\right)-E_{F}\right] / k T}+1}
$$

where $m$ is the effective mass of the electron, $h$ is Planck's constant, and $E_{F}$ is the Fermi energy level measured from the conduction band minimum $E_{c}$ (Fig. 1). Assuming uniform absorption of photons throughout the thin metal film as a consequence of weak absorption (7), the total number of electrons per unit volume $\left(N_{T}\right)$ that are candidates for photoexcitation is

$$
N_{T}=8 \pi\left(\frac{m}{h}\right)^{3} \int_{1 / 2 m \rho^{2}=E_{F}-h \nu}^{\infty} \frac{\rho^{2} d \rho}{e^{\left(1 / 2 m \rho^{2}-E_{F}\right) / k T}+1}
$$

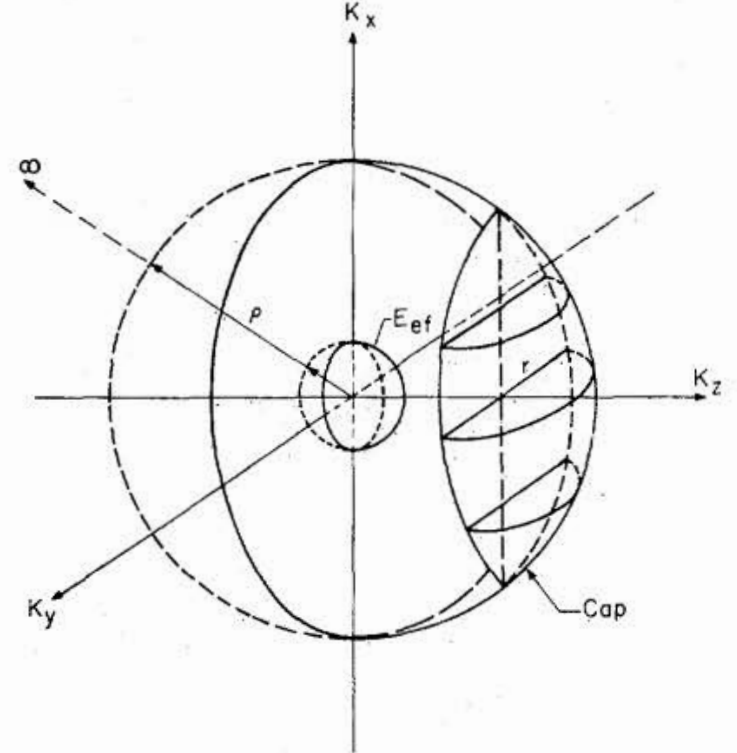

Fig. 3. Three-dimensional $E-K$ relation for the free electron gas of metal; each sphere corresponds to a constant energy surface. Volume of the cap corresponds to the fraction of electrons which can overcome the Schottky barrier height.

where $\rho=\left(u^{2}+v^{2}+w^{2}\right)^{1 / 2}$ and is proportional to the radius of the constant energy sphere indicated in Fig. 3. $h \nu$ is the incident photon energy. In this paper, we depart from the standard Fowler theory of (26) by assuming, as described above, that photoexcitation occurs only from those states belonging to the peak near the Fermi energy. This assumption is justified from an examination of our experimental data and implies that for the metals studied here the density of states is relatively strongly peaked near the Fermi energy. For ease of calculation, we assume that the density of states nevertheless follows a parabolic distribution but with an effective conduction band minimum $E_{c}^{\prime}$ which is close to the Fermi level. We, therefore, refer the Fermi energy to $E_{c}^{\prime}$ so that $E_{e f}=E_{F}-E_{c}^{\prime}$. In the present theory, $N_{T}$ is therefore given as

$$
N_{T}=8 \pi\left(\frac{m}{h}\right)^{3} \int_{1 / 2 m \rho^{2}=E_{F} \sim E_{e f}}^{\infty} \frac{\rho^{2} d \rho}{e^{\left(1 / 2 m \rho^{2}-E_{F}\right) / k T}+1} .
$$

Taking $E_{F} \rightarrow E_{e f}$ ( $E_{c}^{\prime}$ is now the zero of energy), $N_{T}$ will correspond to the volume given by the sphere of Fig. 3 , then

$$
\begin{aligned}
N_{T} & =8 \pi\left(\frac{m}{h}\right)^{3} \int_{0}^{\infty} \frac{\rho^{2} d \rho}{e^{\left(1 / 2 m \rho^{2}-E_{e f}\right) / k T}+1} \\
& =8 \sqrt{2} \pi \frac{(m)^{3 / 2}}{h^{3}}(k T)^{3 / 2} I\left(E_{e f}\right)
\end{aligned}
$$

where

$$
I\left(E_{e f}\right)=\int_{0}^{\infty} \frac{y^{1 / 2} d y}{e^{y-E_{e f} / k T}+1}
$$

and

$$
y=\frac{1}{k T}\left(\frac{1}{2} m \rho^{2}\right)
$$


As explained by Fowler [8], only those electrons whose kinetic energy normal to the metal-Ge interface augmented by $h \nu$ is sufficient to overcome the barrier height $\left(\phi_{B}\right)$ will contribute to the photocurrent in the device. The number of these electrons correspond to the "cap" volume indicated in the constant energy sphere of Fig. 3 and is given as

$$
N_{B}=\int_{1 / 2 m u^{2}=E_{e f}+\phi_{B}-h v}^{\infty} \vec{n}(u) d u
$$

where $\bar{n}(u)$ is the number of electrons per unit volume having a velocity component normal to the metal-Ge interface in the range $u, u+d u$, given by

$$
\begin{aligned}
\vec{n}(u) d u & =2\left(\frac{m}{h}\right)^{3} d u \int_{0}^{\infty} \int_{0}^{2 \pi} \frac{r d r d \theta}{e^{\left[1 / 2 m\left(u^{2}+r^{2}\right)-E_{e f}\right] / k T}+1} \\
& =\frac{4 \pi k T}{m}\left(\frac{m}{h}\right)^{3} \log \left\{1+e^{\left(E_{e f}-1 / 2 m u^{2}\right) / k T}\right\} d u .
\end{aligned}
$$

By making a change of variables and substituting (30) into (29), with $y=\left(h \nu-\phi_{B}-E_{e f}+\frac{1}{2} m u^{2}\right) / k T$, we have that

$$
\begin{aligned}
N_{B}= & \frac{2 \pi k T}{m}\left(\frac{2 k T}{m}\right)^{1 / 2}\left(\frac{m}{h}\right)^{3} \\
& \cdot \int_{0}^{\infty} \frac{\log \left\{1+e^{-y+\left(h \nu-\phi_{B}\right) / k T}\right\}}{\left[y+\left(E_{e f}+\phi_{B}-h \nu\right) / k T\right]^{1 / 2}} d y .
\end{aligned}
$$

To simplify the integral in (31), we consider the region of $h \nu$ near $\phi_{B}\left(h \nu \doteqdot \phi_{B}\right)$; it then becomes a good approximation to neglect $y$ in the denominator of the integrand of (31); therefore,

$$
\begin{aligned}
N_{B} \simeq & \frac{2 \sqrt{2} \pi m^{3 / 2}}{h^{3}} \frac{k^{2} T^{2}}{\left(E_{e f}\right)^{1 / 2}} \\
& \cdot \int_{0}^{\infty} \log \left\{1+e^{-y+\left(h \nu-\phi_{B}\right) / k T}\right\} d y .
\end{aligned}
$$

For

$$
\begin{aligned}
t= & \frac{h v-\phi_{B}}{k T} \geqslant 0 \\
N_{B} \simeq & \frac{2 \sqrt{2} \pi m^{3 / 2} k^{2} T^{2}}{h^{3}\left(E_{e f}\right)^{1 / 2}} \\
& \cdot\left[\frac{1}{2} t^{2}+\frac{\pi^{2}}{6}-\left\{e^{-t}-\frac{e^{-2 t}}{2^{2}}+\frac{e^{-3 t}}{3^{2}}+\cdots\right\}\right] \\
\therefore N_{B} \simeq & \frac{\sqrt{2} \pi m^{3 / 2}}{h^{3}\left(E_{e f}\right)^{1 / 2}}\left[\left(h v-\phi_{B}\right)^{2}+\frac{k^{2} T^{2} \pi^{2}}{3}\right] .
\end{aligned}
$$

So the fraction of the absorbed photons which produce photoelectrons with the appropriate energy and momenta before scattering to contribute to the photocurrent is

$$
F_{e} \simeq \frac{N_{B}}{N_{T}}=\frac{1}{8 E_{e f}^{1 / 2}(k T)^{3 / 2} I\left(E_{e f}\right)}\left[\left(h v-\phi_{B}\right)^{2}+\frac{k^{2} T^{2} \pi^{2}}{3}\right] .
$$

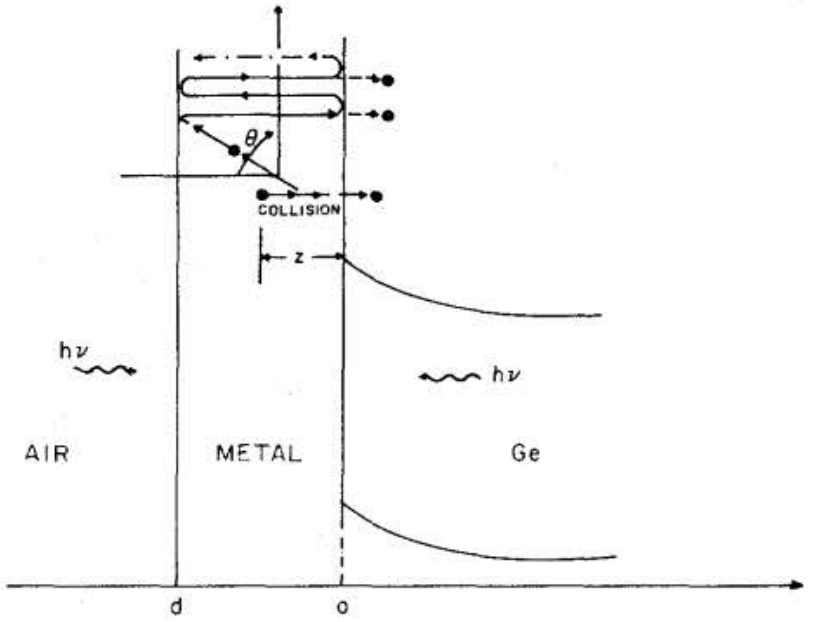

Fig. 4. Schematic diagram of a metal-Ge contact showing the scattering mechanism in the metal film. Solid line indicates the path for multiple scattering, broken lines indicate those electrons which can survive the scattering and be collected by the metal-Ge interface.

\section{Mathematical Formulation for $P_{E}$}

The efficiency of collecting those electrons which have sufficient normal kinetic energy to overcome $\phi_{B}$ will depend on their probability of collisions with the cold electrons and with the two boundary surfaces of the metal. The probability of a collision with the cold electrons is described by a mean free path $L_{e}$. As shown in Fig. 4, the Schottky barrier is illuminated through the front surface of the metal electrode. Since the optical absorption has been assumed uniform throughout the metal thickness, as justified in [7], the average probability of photoexcited electrons arriving at the semiconductor surface without collisions is

$$
\begin{aligned}
p_{t} & =\frac{1}{d} \int_{0}^{d} \exp \left(-z / L_{e}\right) \\
& =\frac{L_{e}}{d}\left[1-\exp \left(-d / L_{e}\right)\right]
\end{aligned}
$$

where $d$ is the metal thickness. Those electrons which have sufficient energy to overcome $\phi_{B}$ after one collision with the cold electrons are oriented with equal probability over the entire half-sphere before reflection by the metal surfaces; their accumulated probability of arrival at the reflection boundary is given by Vickers [11] as

$$
\begin{aligned}
p_{r} & =\frac{1}{d} \int_{0}^{d} \int_{0}^{\pi / 2} \exp \left[-(d-z) / L_{e} \cos \theta\right] \sin \theta d \theta d z \\
& =\frac{1}{d} \int_{0}^{d} \int_{0}^{\infty}\left\{\exp \left[-t(d-z) / L_{e}\right] / t^{2}\right\} d t d z \\
& =\frac{1}{d} \int_{0}^{d} E_{2}\left[(d-z) / L_{e}\right] d z \\
& =\frac{L_{e}}{d}\left[\frac{1}{2}-E_{3}\left(d / L_{e}\right)\right]
\end{aligned}
$$

where $E_{2}$ and $E_{3}$ are exponential integrals. For multiple re- 
TABLE II

Summary OF Results OBTAINED From Figs. 7-10

\begin{tabular}{|c|c|c|c|c|c|c|c|c|c|c|c|c|}
\hline \multirow{2}{*}{$\mathrm{d}(\dot{A})$} & \multicolumn{4}{|c|}{$L_{e}(\AA)$} & \multicolumn{4}{|c|}{$\phi_{B}(e V)$} & \multicolumn{4}{|c|}{$\mathrm{E}_{\mathrm{ef}}(\mathrm{eV})$} \\
\hline & $\mathrm{Au}$ & $\mathrm{Ag}$ & $\mathrm{Cu}$ & $\mathrm{Pb}$ & $\mathrm{Au}$ & $\mathrm{Ag}$ & $\mathrm{Cu}$ & $\mathbf{P b}$ & $\mathrm{Au}$ & Ag & $\mathrm{Cu}$ & $\mathrm{Pb}$ \\
\hline so & 550 & & & & 0.56 & & & & 0.1 & & & \\
\hline 100 & 550 & 570 & 450 & & 0.55 & 0.54 & 0.512 & & 0.1 & 0.152 & 0.11 & \\
\hline 200 & & 570 & & & & 0.54 & & & & 0.152 & & \\
\hline 300 & & 570 & 450 & 55 & & 0.54 & 0.493 & 0.535 & & 0.152 & 0.11 & 0.1 \\
\hline
\end{tabular}

flections, the probability that the capturable electrons can bounce from one metal boundary to another is given as

$$
p=E_{2}\left(d / L_{e}\right) \text {. }
$$

The total accumulated probability that those electrons which have sufficient normal kinetic energy to overcome $\phi_{B}$ will contribute to the photocurrent is then

$$
\begin{aligned}
P_{E} & =p_{t}+p_{r}\left(1+p+p^{2}+p^{3}+\cdots\right) \exp \left(-d / L_{e}\right) \\
& =\frac{L_{e}}{d} U\left(\frac{d}{L_{e}}\right)
\end{aligned}
$$

where

$$
U\left(\frac{d}{L_{e}}\right)=\frac{d}{L_{e}}\left\{p_{t}+\frac{p_{r}}{(1-p)} \exp \left(-\frac{d}{L_{e}}\right)\right\}
$$

and

$$
U\left(\frac{d}{L_{e}}\right) \simeq\left[1-\exp \left(-\frac{d}{L_{e}}\right)\right]^{1 / 2} .
$$

Therefore, the external quantum efficiency $\left(\eta_{0}\right)$ associated with internal photoemission can be concluded from (1), (24), (34), and (38) to be

$$
\eta_{0} \simeq A_{T} \frac{L_{e}}{d} U\left(\frac{d}{L_{e}}\right) \frac{\left[\left(h \nu-\phi_{B}\right)^{2}+(k T \pi)^{2} / 3\right]}{8 E_{e f}^{1 / 2}(k T)^{3 / 2} I\left(E_{e f}\right)} .
$$

\section{Numerical Method}

A computer program, which incorporates (1) to (24), (40), and (41), is run interactively to obtain the optimal agreement with the experimental results. The optical parameters used in the above calculation are assumed to be relatively constant over the wavelength range under consideration, and are in good agreement with results reported in [7], [10], [12]-[14]. $\phi_{B}$ is measured independently and $L_{e}$ and $E_{e f}$ are adjusted to obtain the best fit to experimental data; their final values are summarized in Table II and will be discussed in the following sections.

\section{EXPERIMENTAL PRocedure}

\section{A. Fabrication}

n-type germanium wafers of (111) orientation with 0.1-1.0 $\Omega \cdot \mathrm{cm}$ resistivity were used in this study; these were etched in CP-4A solution for $8 \mathrm{~min}$ prior to metal evaporation. Ohmic contacts were made by evaporating a thick film of high purity tin at the back of the slices. All metal evaporations were per- formed in a liquid-nitrogen baffled oil-diffusion system with base pressure not greater than $8 \times 10^{-6}$ torr. Deposition of the metal electrodes was through an "out-of-contact" mask to give several circular contacts ( $a r e a \simeq 8.11 \times 10^{-3} \mathrm{~cm}^{2}$ ) on each wafer. Metal thicknesses were monitored by an Edwards F.T.M. 2 film thickness monitor; these thicknesses were in the range from 50 to $1000 \AA$.

\section{B. Electrical Measurements $\langle C-V, I-V\rangle$ in Darkness}

Current-voltage $(I-V)$ characteristics were plotted using a variable dc power supply and $x-y$ recorder with the device in darkness. Capacitance-voltage data $(C-V)$ at $1 \mathrm{MHz}$ was measured using a Boonton Model 72 B capacitance meter again under dark conditions. $C-V$ data were then transcribed to $1 / C^{2}$ versus $V$ plots to obtain the barrier heights $\left(\phi_{B}\right)$ and doping density $\left(N_{d}\right)$.

\section{Photoresponse Measurements in the Infrared Region $1 \mu m<\lambda \lesssim 2 \mu m$}

A SPEX model 1670 grating monochromator with resolution $\sim 10^{-4} \mathrm{eV}$ was used to generate the optical signal in the range $1 \mu \mathrm{m}<\lambda \lesssim 2 \mu \mathrm{m}$. A Corning RG $1000 \mathrm{D}$ filter was used to eliminate radiation due to higher order diffraction. The optical power density $\left(p_{i}\right)$ on the device at different $\lambda$ was calibrated by an Epply linear thermopile (No. 15043). The short-circuit $(V=0)$ photogenerated current $\left(I_{s c}\right)$ of the device was measured by a GR 1807 dc nanoammeter. The quantum efficiency for $V=0$ is calculated as

$$
\eta_{0}=\left(\frac{I_{s c}}{q p_{i} a} \frac{1.24}{\lambda} \times 100\right) \text { percent }
$$

where $a$ is the device area.

\section{RESUltS AND Discussion}

The results for the $I-V$ and $C-V$ characteristics for the metal-Ge Schottky barriers have been discussed in detail in our previous work [5], [6]. We summarize the typical results for those devices used for the present analysis in Table III. These devices have demonstrated very nearly ideal $I-V$ characteristics with $n$ values very close to unity. $\phi_{B}$ 's determined from $I-V$ and $C-V$ measurement are in good agreement within an error of $\pm 0.03 \mathrm{eV}$. Shown in Fig. 5 are some typical data obtained for internal photoemission for a set of devices with metal thickness $\leqslant 350 \AA$.

Shown in Fig. 6 is $\eta_{0}^{1 / 2}$ versus $h \nu$ (expanded scale) for $\mathrm{Cu}-\mathrm{Ge}$ Schottky barriers at three different metal electrode thick- 
TABLE III

Summary of Schottky Barrier Height $\left(\phi_{B}\right)$ Measured by Four DIFFERENT METHODS

\begin{tabular}{|c|c|c|c|c|c|c|c|c|c|c|c|c|c|c|c|c|}
\hline \multirow{3}{*}{$\begin{array}{l}\text { METAL } \\
\text { THICKNESS } \\
\text { (d) } \AA\end{array}$} & \multicolumn{16}{|c|}{$\phi_{B}(e V)$} \\
\hline & \multicolumn{4}{|c|}{$\begin{array}{c}\text { FORWARD } \\
I-V\end{array}$} & \multicolumn{4}{|c|}{$\begin{array}{c}\text { REVERSE } \\
I-V\end{array}$} & \multicolumn{4}{|c|}{$c-v$} & \multicolumn{4}{|c|}{ PHOTORESPONSE } \\
\hline & Au & $\mathrm{Ag}$ & $\mathrm{Cu}$ & $P b$ & $\mathrm{Au}$ & $\mathrm{Ag}$ & $\mathrm{Cu}$ & $\mathrm{Pb}$ & $\mathrm{Au}$ & $\mathrm{Ag}$ & $\mathrm{Cu}$ & $\mathrm{Pb}$ & $A u$ & $A_{g}$ & $\mathrm{Cu}$ & $\mathrm{Pb}$ \\
\hline 50 & 0.55 & & & & 0.55 & & & & 0.57 & & & & 0.56 & & & \\
\hline 100 & 0.565 & 0.55 & 0.527 & & 0.545 & 0.54 & 0.5 & & 0.54 & 0.53 & 0.51 & & 0.55 & 0.54 & 0.512 & \\
\hline 200 & & 0.55 & & & & 0.54 & & & & 0.53 & & + & & 0.54 & & \\
\hline 300 & & 0.56 & 0.5 & 0.55 & & 0.535 & 0.5 & 0.53 & & 0.525 & 0.48 & 0.525 & & 0.54 & 0.493 & 0.535 \\
\hline 500 & & & 0.499 & & & & 0.5 & & & & 0.483 & & & & 0.49 & \\
\hline 750 & & & 0.512 & & & & 0.51 & . & & 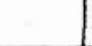 & 0.5 & & & & 0.49 & \\
\hline
\end{tabular}

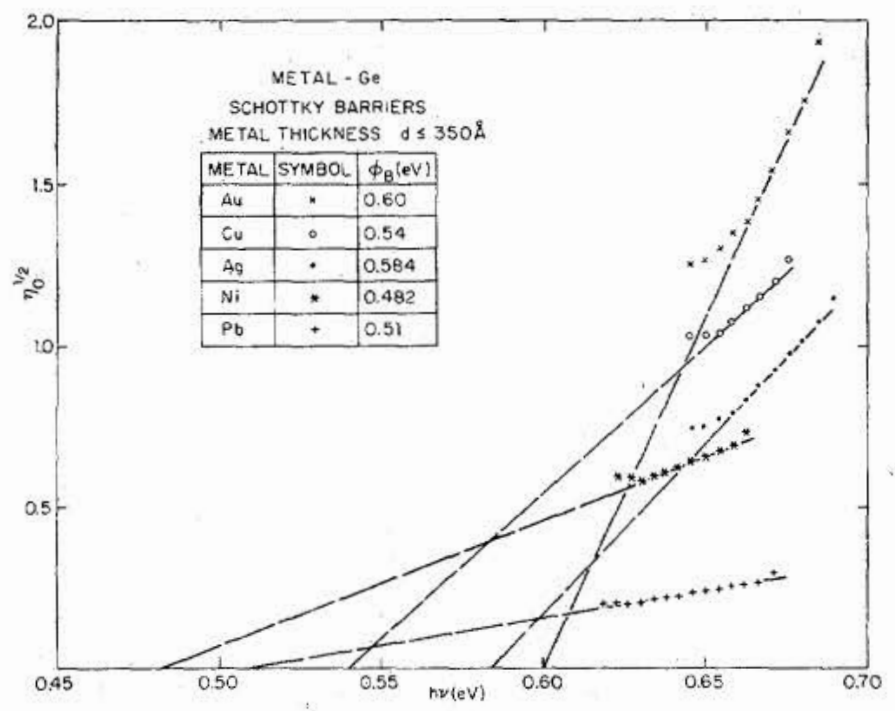

Fig. 5. Raw photoresponse data for a set of metal-Ge Schottky barriers with metal thickness $d \leqslant 350 \AA$. $\phi_{B}$, in the table of the insert, is obtained by the empirical relation $\eta_{0}^{1 / 2} \propto\left(h \nu-\phi_{B}\right)$.

nesses. These data are well fitted by straight lines which intersect the $h \nu$ axis at the same threshold $\left(\phi_{B}=0.49 \mathrm{eV}\right)$. This demonstrates the tendency of photoresponse measurements to yield more consistent $\phi_{B}$ 's for different metal electrode thicknesses than the $I-V$ and $C-V$ methods.

In Fig. 7, we show the results of our analysis for a $\mathrm{Pb}-\mathrm{Ge}$ Schottky barrier with $d=300 \AA$ and $\phi_{B}=0.535 \mathrm{eV}$. Solid curves are the theoretical calculations for different values of hot electron mean free path $\left(L_{e}\right)$. Good agreement with experimental results is obtained at $L_{e}=55 \AA$, and $E_{e f}=0.1 \mathrm{eV}$. Shown in Fig. 8 is the analysis of Au-Ge Schottky barriers with $d=50$ and $100 \AA$, respectively. Good agreement of theory with experimental data is obtained at $L_{e}=550 \AA$ and $E_{\text {ef }}=0.1 \mathrm{eV}$ for both devices.

Fig. 9 shows a similar analysis for $\mathrm{Ag}-\mathrm{Ge}$ Schottky barriers with $d=100,200$, and $300 \AA$. All three devices demonstrate good agreement with theory for the same $L_{e}(570 \AA), E_{\text {ef }}$ $(0.152 \mathrm{eV})$, and $\phi_{B}(0.54 \mathrm{eV})$. Shown in Fig. 10 is the analysis for $\mathrm{Cu}-\mathrm{Ge}$ Schottky barriers with $d=100$ and $300 \AA$. Again good agreement of theory with experimental data is obtained at $L_{e}=450 \AA$ and $E_{e f}=0.11 \mathrm{eV}$ for both devices. The $300 \AA$

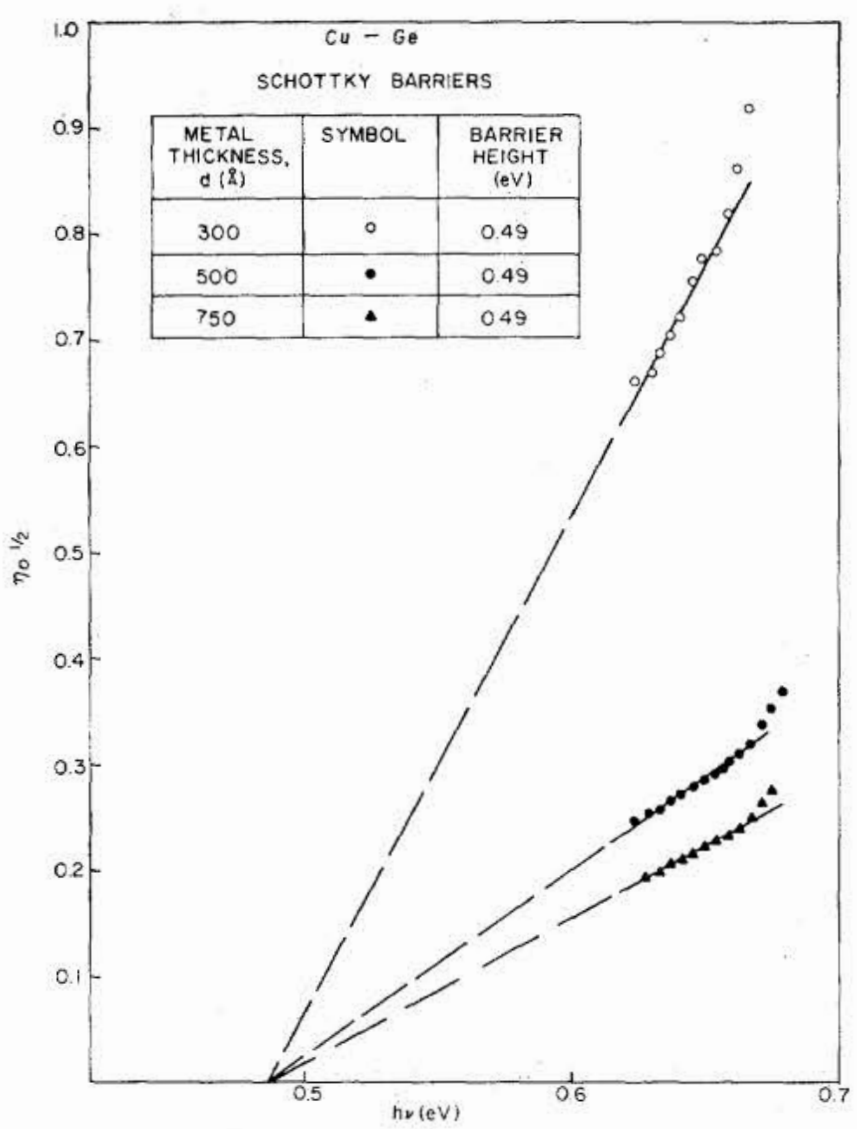

Fig. 6. Experimental results of $\eta_{0}^{1 / 2}$ versus $h \nu$ for $\mathrm{Cu}-\mathrm{Ge}$ Schottky barrier at three different metal thicknesses. All three devices show good agreement for $\phi_{B}$ and their results are fit well by three straight lines.

$\mathrm{Cu}-\mathrm{Ge}$ contact of Fig. 10 is the same as the $300 \AA \mathrm{Cu}-\mathrm{Ge}$ contacts of Fig. 6. $\phi_{B}$ from Fig. 10 for this device is approximately $0.49 \mathrm{eV}$.

It is apparent from the present experimental results that the photoemission occurs from a narrow range of energy on the order of a fraction of an electron volt below the Fermi level; this is consistent with a density of states in the thin metal film which shows a narrow peak in this energy range. The work of Berglund and Spicer [9] on thick copper and silver films indicates that narrow peaks of this nature may be present near the Fermi energy and that broader peaks (Van Hove singularities) 


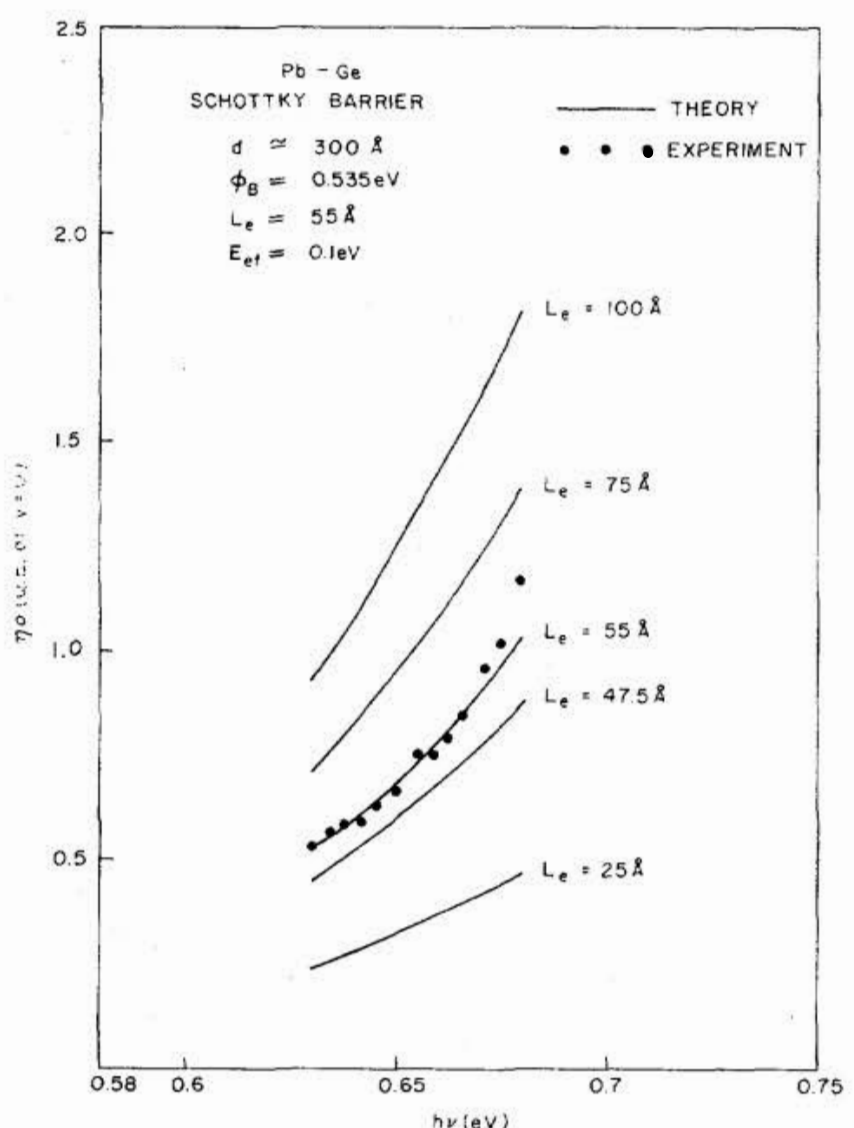

Fig. 7. $\eta_{0}$ versus $h \nu$ for a $\mathrm{Pb}-\mathrm{Ge}$ Schottky barrier at $d=300 \AA$. Solid curves represent computer caiculations for different mean free paths $\left(L_{e}\right)$. The best fit to the experimental data is obtained at $L_{e}=55 \AA$, $\phi_{B}=0.535 \mathrm{eV}$, and $E_{e f}=0.10 \mathrm{eV}$.

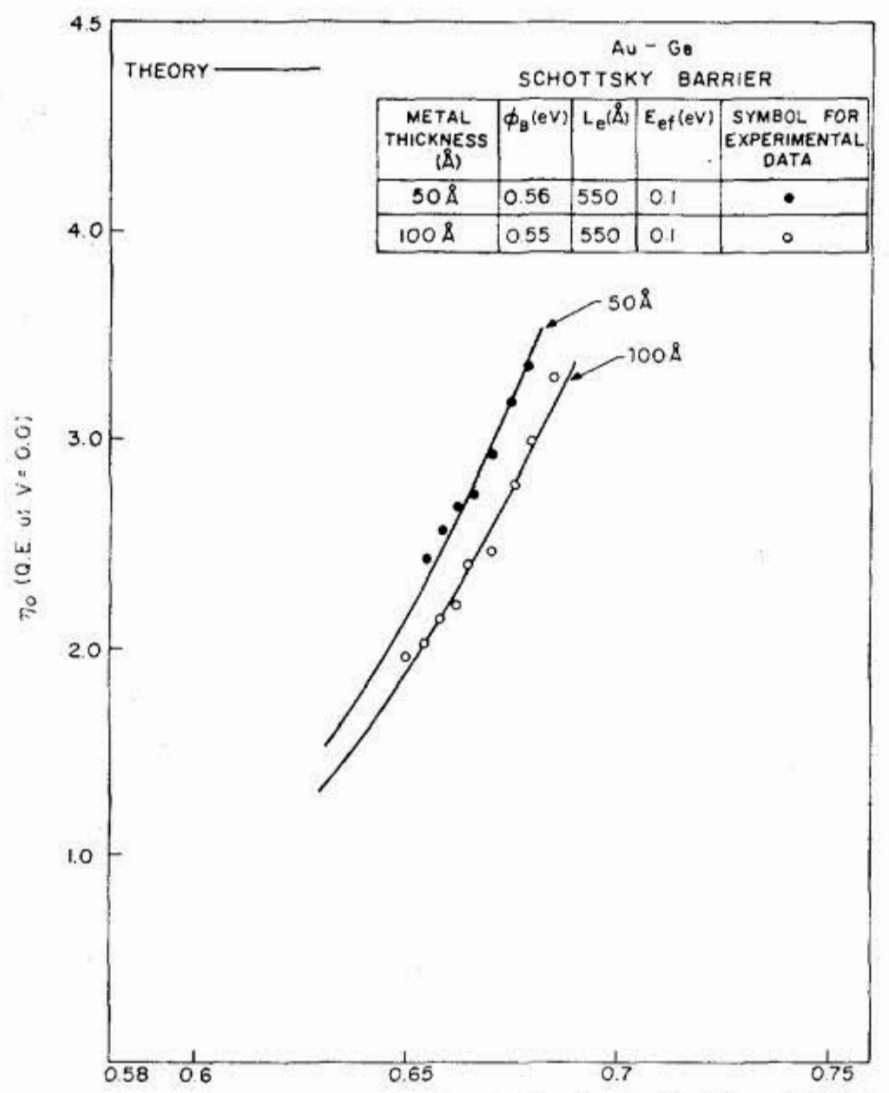

Fig. 8. $\eta_{0}$ versus $h \nu$ for Au-Ge Schottky barrier at $d=50$ and $100 \AA$. Good agreement of theory with experimental data is obtained at $L_{e}=550 \mathrm{~A}$ and $E_{e f}=0.1 \mathrm{eV}$.

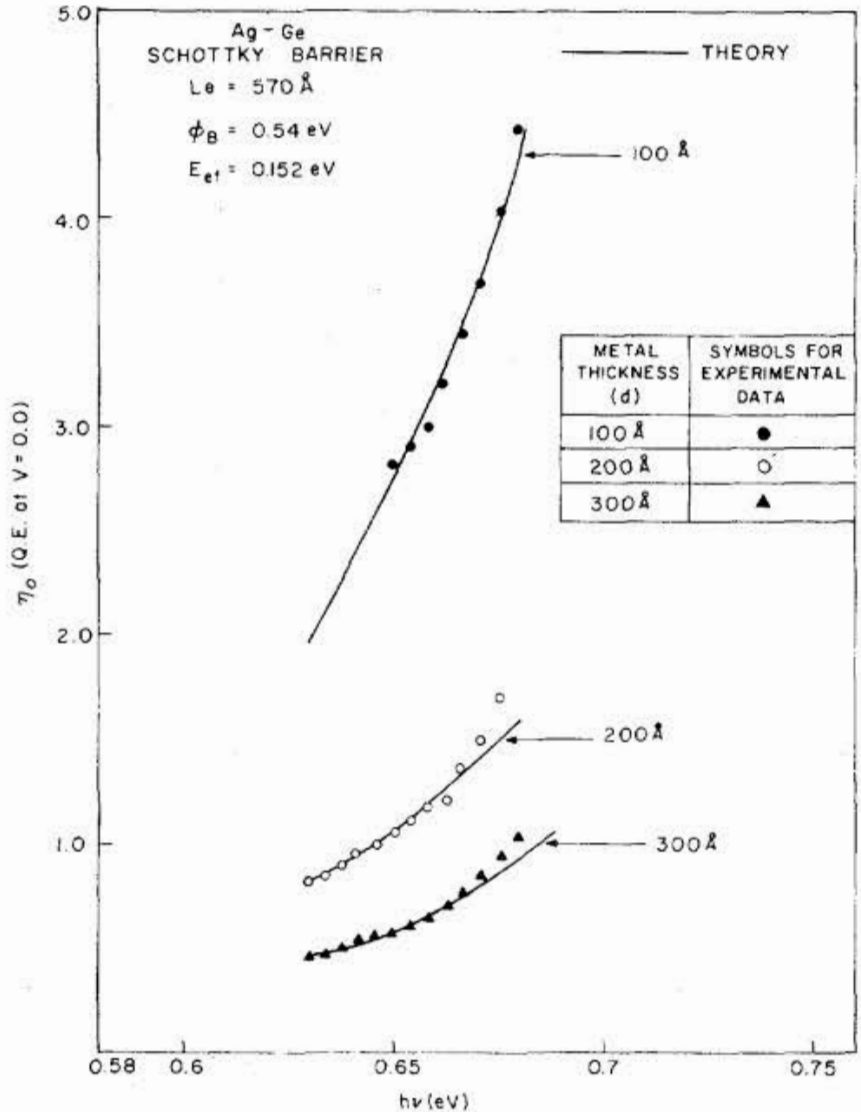

Fig. 9. $\eta_{0}$ versus $h v$ for Ag-Ge Schottky barrier at $d=100,200$, and $300 \AA$. Good agreement of theory with experimental data is obtained at $L_{e}=570 \AA, E_{e f}=0.152 \mathrm{eV}$, and $\phi_{B}=0.54 \mathrm{eV}$, for all three devices.

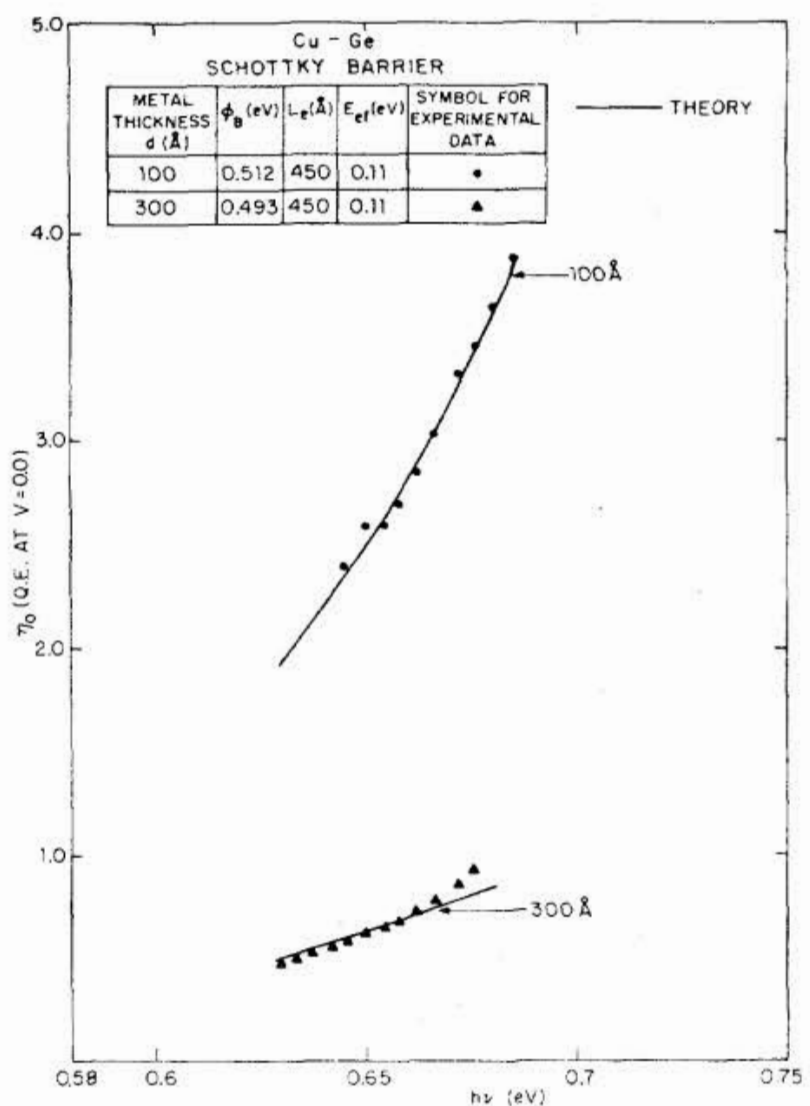

Fig. 10. $\eta_{0}$ versus $h \nu$ for $\mathrm{Cu}-\mathrm{Ge}$ Schottky barrier at $d=100$ and $300 \AA$. Good agreement of theory with experimental data is obtained at $L_{e}=$ $450 \AA$ and $E_{\text {ef }}=0.11 \mathrm{eV}$. 
are present at energies (several electron volts) well below the Fermi level. These lower lying states, however, are not accessible to photoemission for the range of energies $(0.6-0.7 \mathrm{eV})$ employed here.

\section{CONCLUSION}

The quantum efficiency of thin metal film-Ge Schottky barriers associated with the photoemission of electrons from the metal can be explained by considering: 1) those photoexcited electrons in the metal which have sufficient kinetic energy normal to the metal-Ge interface to overcome $\phi_{B}$, and induced by uniform absorption of the incident photons, 2) multiple scattering of hot electrons in the metal, and 3) multiple reflection of transmitted photons in the Ge substrate. The theoretical model presented here is consistent with the experimental data insofar as the hot electron mean free path $\left(L_{e}\right)$ and effective Fermi-energy $\left(E_{e f}\right)$ deduced from the comparison of theory and experiment is independent of metal thickness for each type of metal-Ge contact. $E_{\text {ef }}$ is quite small $(\simeq 0.1 \mathrm{eV})$ which implies that the $0.6-$ $0.7 \mathrm{eV}$ photons excite electrons from the near-vicinity of the Fermi energy level, in qualitative agreement with the density of states distributions of Berglund and Spicer [9]. Internal photoemission in thin metal films is relatively well understood by the present theoretical description which is expected to enable the optimization of Schottky barrier detectors operating in this mode. This mechanism provides an extension to longer wavelength operation (beyond the normal cutoff associated with the energy gap of the semiconductor) of metal-Ge Schottky barrier quantum detectors.

\section{ACKNOWLEDGMENT}

We wish to thank Prof. E. S. Yang and W. Hwang, as well as K. K. Ng and P. Panayotatos for stimulating discussions.

\section{REFERENCES}

[1] K. S. Chaplin and G. Eisenstein, "Infrared response of lightly doped Schottky diodes," Appl. Phys. Lett,, vol. 31, p. 221, Aug. 1977.

[2] A. A. Gutkin, M. V. Dmitriev, and V. M. Khair, "Photosensitivity of Au-n-Si surface-barrier diodes in the spectral range 1-6 eV," Sov. Phys. Semicond., vol. 11, p. 290, Mar. 1977.

[3] F. D. Shepherd, Jr. and A. C. Yang, "Silicon Schottky retinas for infrared imaging," International Electron Devices Meeting (IEDM) Technical Digest, pp. 310-313, Dec. 1973.

[4] H. D. Law, L. R. Tomasetta, K. Nakano, and J. S. Harris, "1.0$1.4 \mu \mathrm{m}$ high-speed avalanche photodiodes," Appl. Phys. Lett., vol. 33 , p. 416 , Sept. 1978.

[5] E.Y.Chan and H. C. Card, "Optoelectronic properties of metal-Ge Schottky barrier quantum detectors," International Electron Devices Meeting (IEDM) Technical Digest, pp. 653-656, Dec. 1978.

[6] -, "Infrared optoelectronic properties of metal-Ge Schottky barriers," IEEE Trans. Electron Devices, vol. ED-27, pp. 78-83, Jan. 1980.

[7] - "Near infrared interband transitions and optical parameters of metal-Ge contacts," Appl. Opt., to be published.

[8] R. H. Fowler, "The analysis of photoelectronic sensitivity curves for clean metals at various temperatures," Phys. Rev., vol. 38, p. 45, July 1931 .

[9] C. N. Berglund and W. E. Spicer, "Photoemission studies of copper and silver: Theory and experiment," Phys. Rev., vol. 136, pp. A $1030-1064$, Nov. 1964.

[10] O. S. Heavens, "Optical properties of thin films," Rep. Progress in Phys., Inst. Phys. and the Phys. Soc., London, England, 23, 1960 , pp. 1-65.
[11] V. E. Vickers, "Model of Schottky barrier hot-electron-mode photodetection," Appl. Opt., vol. 10, pp. 2190-2192, Sept. 1971.

[12] L. S. Schulz, "The optical constants of silver, gold, copper and aluminum: I. The absorption coefficient $\mathrm{k}$; II. The index of refraction n," J. Opt. Soc. Amer., vol. 44, pp. 357-370, May 1974.

[13] L. G. Schulz, "An experimental confirmation of the drude free electron theory of the optical properties of metals for silver, gold and copper in the near infrared," J. Opt. Soc. Amer., vol. 44 , p. 540 , May 1954

[14] H. M. O'Bryan, "The optical constants of several metals in vacuum," J. Opt. Soc. Amer., vol. 26, p. 122, Mar. 1936.

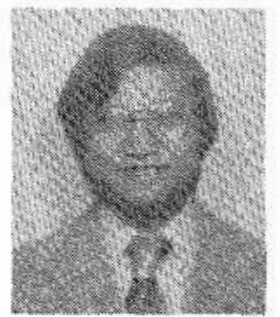

Eric Y. Chan (M'76) was born in Hong Kong, China, on November 23, 1949. He received the B.S. degree with honors from the University of California, Berkeley, in 1973, and the M.S. and Ph.D. degrees from Columbia University, New York, NY, in 1975 and 1979 , respectively.

$\mathrm{He}$ is presently a member of the Research Staff of the Western Electric Engineering Research Center, Princeton, NJ. His research interests are solid-state optoelectronic devices and fiber-optic communication.

Dr. Chan is a member of the American Physical Society, Sigma Xi, and Eta Kappa $\mathrm{Nu}$.

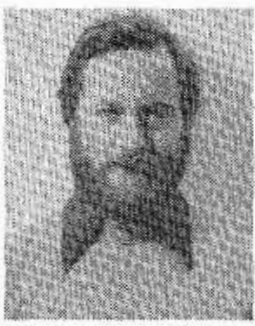

Howard C. Card (S'68-M'72) was born in Winnipeg, Manitoba, Canada, on March 30, 1947. $\mathrm{He}$ received the B.Sc.E.E. and M.Sc. degrees from the University of Manitoba, Manitoba, Canada, and the Ph.D. degree from the University of Manchester Institute of Science and Technology, in 1968, 1969, and 1971, respectively.

From 1971 to 1974 he was employed at UMIST as a Mullard Research Fellow, engaged in work on nonvolatile MOS memory transistors and metal-semiconductor contacts. From 1974 to 1975 he was with the University of Waterloo, Ontario, Canada, involved in functional modeling of semiconductor devices. He is presently an Associate Professor at Columbia University, New York, NY, and a Consultant to IBM Watson Research Center, with research interests in the physics of semiconductor devices, interface phenomena, VLSI integrated circuits, optical electronics, and photovoltaic energy conversion.

Dr. Card is a member of the (European) Institute of Physics, the American Physical Society, the Optical Society of America, Sigma Xi, Eta Kappa Nu, and the New York Academy of Sciences.

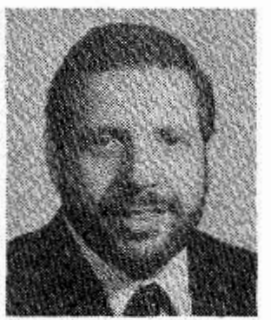

Malvin C. Teich (S'62-M'66-SM'72) was born in New York City, NY, on May 4, 1939. He received the S.B. degree in physics from the Massachusetts Institute of Technology, Cambridge, MA, in 1961, the M.S. degree in electrical engineering from Stanford University, Stanford, CA, in 1962, and the Ph.D. degree in quantum electronics from Cornell University, Ithaca, NY, in 1966. While at M.I.T. he received the Frank W. and Carl S. Adams Memorial Scholarship, and at Cornell University he received the James Clerk Maxwell Fellowship and a Ford Foundation Grant.

He has held summer research positions at the Motorola Corporation, Rockwell International's Space and Information Systems Division, and the Mitre Corporation in 1959, 1961, and 1963, respectively. In the 
summer of 1960 he participated in a student technical exchange program at N.V. Philips Gloeilampenfabrieken in Eindhoven, The Netherlands. In 1966 he joined the M.I.T. Lincoln Laboratory, Lexington, MA, where he was engaged in work on coherent infrared detection and modulation. In 1967 he became a member of the faculty in the Department of Electrical Engineering, Columbia University, New York, NY, where he is now teaching and pursuing his research interests in the areas of optical and infrared detection, lightwave communications, and sensory perception. He is also currently serving as Chairman of the Department, as a member of the faculty in the Department of Applied Physics and Nuclear Engineering, and as a member of the Columbia
Radiation Laboratory. He has authored or coauthored 75 technical publications and holds one patent.

Dr. Teich is a member of Sigma Xi, the American Physical Society, the Optical Society of America, the Acoustical Society of America, the Society of Photo-Optical Instrumentation Engineers, the American Society for Engineering Education, the Society for Neuroscience, and the New York Academy of Sciences. He is a member of the Editorial Advisory Panel for Optics Letters. In 1969 he was the recipient of the IEEE Browder J. Thompson Award for his paper "Infrared Heterodyne Detection," and in 1973 was appointed a Fellow of the John Simon Guggenheim Memorial Foundation.

\title{
Notes and Lines
}

\section{Correction to "Coherent Optical Processing: Another Approach"}

\author{
In the table of contents of the December 1979 issue, the \\ term "Invited Paper" was inadvertently omitted after the title \\ of the above paper. ${ }^{1}$ \\ ${ }^{1}$ B. D. Guenther, C. R. Christensen, and J. Upatnieks, IEEE J. Quan- \\ tum Electron., vol. QE-15, pp. 1348-1362, Dec. 1979.
}

\section{Book Reviews}

Acousto-Optics-J. Sapriel (Translated by S. Francis and B. Kelly). (New York: Wiley, 1979, 126 pp., \$20.00). Reviewed by M. D. Fox.

This slender but ambitious monograph attempts to unify in a single volume a large number of topics, each of which could easily occupy textbooks in their own right. These topics include piezoelectricity, elasticity, photoelastic effects, bulk and surface acoustic wave propagation, acoustooptic interactions, and experimental determination of acoustic and acoustooptic properties of materials. The aim is to provide both a reference work for researchers and an introductory textbook for seniors or beginning graduate students. Given the broad sweep of the objectives and the small size of the volume, perhaps the author should be forgiven for not entirely achieving his goals.

The unifying theme for tying together the diverse physical phenomena described is a powerful tensor notation which is introduced in the first chapter. In succeeding chapters, elasticity theory, propagation of plane acoustic waves in piezoelectric and nonpiezoelectric media, and photoelastic properties of crystals are introduced using this tensor approach. Piezoelectricity is rather neatly introduced as an extension of the elastic description for nonpiezoelectric materials. These introductory theoretical chapters pave the way for the final three chapters discussing diffraction of light by acoustic waves, acoustooptic deflectors and modulators, and the determina-

The reviewer is with University of Connecticut, Storrs, CT 06268. tion of acoustic and acoustooptic characteristics of materials by experimental methods. The last three chapters differ markedly from the first five by emphasizing experimental results, and rarely employ the tensor notation introduced in the first five chapters. A final conclusion section is very brief.

In trying to cover so much ground, some topics are necessarily treated rather superficially. In the discussion of piezoelectric transducers, for example, the author presents a discussion of power transfer into transducers, but neglects the more important problem of maximizing bandwidth. He also omits mention of the large body of engineering literature on the subject of transducer design. The discussion on the determination of elastic and piezoelectric constants by resonance measurements is very brief and ignores such significant practical problems as variation of dielectric constant with frequency, and the existence of spurious resonances, which are particularly prominent in plates vibrating in air.

Overall, the author was probably wise to emphasize a phenomenological description of the various effects covered, and to avoid delving into the microscopic theories, whose validity may be questionable. However, by introducing a very general tensor notation, many of the theoretical discussions are so general as to be very abstract and difficult for the introductory student to visualize. Thus, despite the solved exercises, it seems to me that the book would be difficult to use as a text. As a reference for those working in the field, it does bring together a large body of material in a concise way under a unified notation. 